\title{
Harmonic Elimination and Optimization of Stepped Voltage of Multilevel Inverter by Bacterial Foraging Algorithm
}

\author{
Reza-Salehi*, Behrooz-Vahidi ${ }^{\dagger}$, Naeem-Farokhnia* and Mehrdad-Abedi*
}

\begin{abstract}
A new family of DC to AC converters, referred to as multilevel inverter, has received much attention from industries and researchers for its high power and voltage applications. One of the conventional techniques for implementing the switching algorithm in these inverters is optimized harmonic stepped waveform (OHSW). However, the major problem in using this technique is eliminating low order harmonics by solving the nonlinear and complex equations. In this paper, a new approach called the "bacterial foraging algorithm" (BFA) is employed. This algorithm eliminates and optimizes the harmonics in a multilevel inverter. This method has higher speed, precision, and convergence power compared with the genetic algorithm (GA), a famous evolutionary algorithm. The proposed technique can be expanded in any number of levels. The purpose of optimization is to remove some low order harmonics, as well as to ensure the fundamental harmonic retained at the desired value. As a case study, a 13-level inverter is chosen. The comparison results by MATLAB software between the two optimization methods (BFA and GA) have shown the effectiveness and superiority of BFA over GA where convergence is desired to achieve global optimum.
\end{abstract}

Keywords: Multilevel Inverter, Harmonic Elimination, Bacterial Foraging Algorithm, Genetic Algorithm

\section{Introduction}

Increasing growth in consumption and variety of loads has been observed over time and with the expansion in the electrical industry. In recent years, applications of high voltage and high power equipment have grown, such that the power levels of equipment have reached the megawatt range. Today, creating a direct connection of single semiconductor switch to a system with medium voltage grids $(2.3,3.2,4.16$, and $6.9 \mathrm{kV})$ is difficult to achieve [1]. This gap has to be resolved because of the significance of some loads and their sensitivity to voltage sag and swell, harmonic disturbances, and failing and de-rating of other loads resulting from existing disturbances, such as voltage and current. Inverters, such as DC to AC converters, are notable sources of disturbance.

Inverters are widely used in many applications, such as flexible AC transmission system (FACTS) equipment [2], high voltage direct current (HVDC) lines [3], and electrical drives [4]. Different methods have been suggested for improving the quality and performance of inverters. The first approach is by using various switching strategies, such as sinusoidal or "subharmonic" natural pulse width modulation (SPWM), selective harmonic-eliminated pulse width modulation (SHE PWM), space-vector modulation (SVM), optimized harmonic-stepped waveform (OHSW) [5-6], and optimal minimization of THD (OMTHD) [7]. The second

$\dagger \quad$ Corresponding Author: Dept. of Electrical Engineering, Amikabir University of Technology, Tehran, Iran (vahidi@aut.ac.ir)

* Dept. of Electrical Engineering, Amikabir University of Technology, Tehran, Iran (hreza_salehi@aut.ac.ir, farokhnia@aut.ac.ir, abedi@aut.ac.ir )

Received: April 14, 2010; Accepted: July 23, 2010 approach is by using low pass filters in the output of inverters to remove high order harmonics. Finally, the third approach is by applying multilevel structures in order to reduce harmonics and THD, as well as to omit filters in high power applications. Conventional structures of multilevel inverters deal with diode-clamped [8], flying capacitor [9], and cascaded multilevel inverters with separate DC sources (SDCS) [10]. The OHSW technique has been used in multilevel inverters, as evidenced in literature. In this method, the goal is to conduct potential elimination of low order harmonics; when this goal cannot be obtained, the highest possible harmonics optimization is desired. In this approach, n-1 low order harmonics from the fifth order (i.e., given that triple harmonics in three-phase systems, even harmonics from a half-wave symmetric, are automatically eliminated) can be eliminated and the fundamental component can be satisfied by solving the $\mathrm{n}$ equations. However, the major problem encountered by the OHSW technique is solving a set of nonlinear equations.

Several methods have been suggested for solving these equations; each of them has some advantages and disadvantages that can categorized into two sets.

The first group attempts to solve the equations. In [11], the Newton-Raphson (N-R) method is exploited to solve the equations. Iterative methods mainly depend on an initial guess; in addition, divergence problems are likely to occur, especially for high numbers of inverter levels [12]. Although the N-R method is conveniently fast, it can only find one set of solutions. Furthermore, all roots can be obtained using MATLAB function fsolve [13]. This function, based on the Gauss-Newton method, provides a mix of quadratic and cubic line search methods. Meanwhile, the mathematical theory of resultants can be used in [14]. Although this method is complicated and time-consuming 
[11], it finds all possible solutions with the exact range of modulation index $(\mathrm{M})$ at which the solutions exist. For any changes in voltage level or input DC voltage, new expressions are required. By increasing the levels of multilevel inverters, the equation set tends to obtain high order polynomials, which have narrow feasible solution space that are difficult to solve [15]. Homotopy algorithm is another applied approach [16] to determine one set of solutions. Overall, all methods included in the first group do not suggest any optimum solutions for an infeasible M. However, in many applications, working in a continuous range of $\mathrm{M}$ is inevitable. Thus, the second group methods have been presented in literature.

The second group of methods can find solutions that deal with eliminating completely low order harmonics. When no feasible solution exists, the second group introduces optimum angles. In these cases, all evolutionary search algorithms can be regarded as suitable choices. These approaches are simple and applicable for problems that deal with any number of levels; that is, without extensive derivation of analytical expressions, both for eliminating and minimizing harmonics. However, these methods are not as fast as the first group of methods. GA, a wellknown evolutionary algorithm, has been widely used in literature [12, 15]. In [5], a novel powerful optimization algorithm, PSO, which shows significant privileges over binary GA in terms of precision and convergence rate, has been presented. Additionally, in [17], GA and PSO are both utilized to find a suitable initial guess for N-R. This N-R enhances the GA and PSO precisions in terms of reducing fitness values. In this group set, continues GA (CGA) has been recognized as an efficient method.

In this paper, "bacterial foraging algorithm" (BFA) is applied to minimize some specific low order harmonics, as well as to satisfy the desired fundamental component. Results, including the effect of running times on the probability of reaching a global solution for some low-level inverters, are compared with those obtained by GA. Results confirm the effectiveness of the proposed algorithm and its superiority over CGA in terms of convergence rate and probability to reach the global minimum.

\section{Multilevel Inverters}

\subsection{Multilevel Inverter Topologies}

Three different topologies are proposed for multilevel inverters. Fig. 1 shows the cascaded multilevel inverter with separated DC sources.

The multilevel inverter (Fig. 1) has many advantages, such as circuit layout flexibility, absence of extra clamping diodes or voltage balancing capacitors, and easy adjustment of the number of output voltage levels by adding or reducing full-bridge cells. It also has the least number of components compared with other inverters. Switching devices turn on and off only once per cycle to overcome the switching loss problem [18].

Fig. 2 provides additional details on the multilevel inverter circuit.

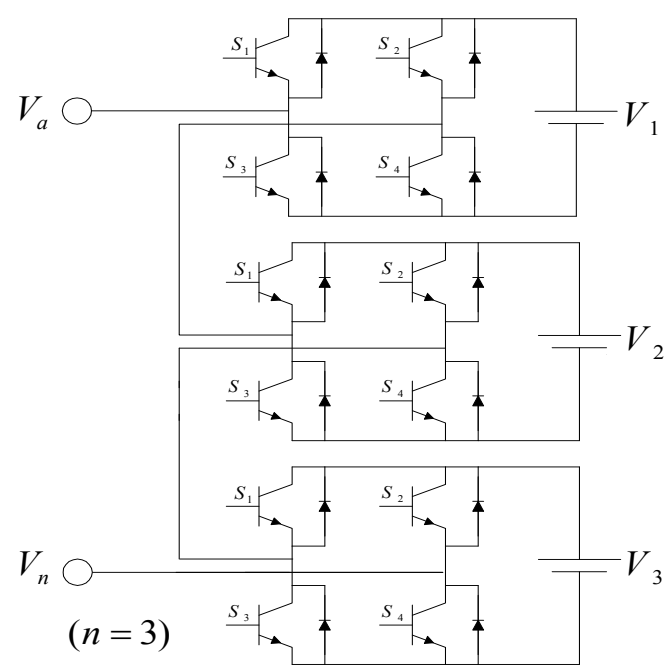

Fig. 1. Cascaded multilevel inverter with separated DC sources.

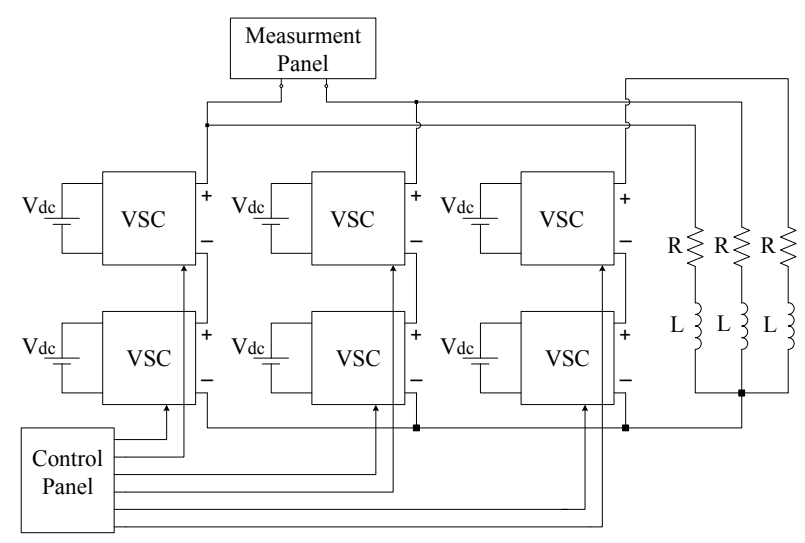

Fig. 2. Schematic of the implemented multilevel inverter.

Due to great demands for medium-voltage high-power inverters, the cascaded inverter has since drawn tremendous interest. This configuration recently became very popular in the field of AC power supply; it also allows for adjustable speed drive applications. In back-to-back inverter applications, it is somewhat impossible to use multilevel inverters using cascaded inverters with Separate DC source because a short circuit is introduced when two backto-back inverters are not synchronously switched [18]. This multilevel inverter is made of several full-bridge inverters. The AC output of each of the different levels of full-bridge inverters are connected in series, such that the synthesized voltage waveform corresponds to the sum of the inverter outputs. However, all of the mentioned inverters can produce multilevel voltage or current, as shown in Fig. 3.

\subsection{Optimized Harmonic Stepped-Waveform Tech- nique (OHSW)}

Fig. 3 shows a seven-level inverter. The variables $\theta_{1}, \theta_{2}$, and $\theta_{3}$ should be determined. The variables $V_{1}, V_{2}$, and $V_{3}$ are the voltages of the DC sources from Fig. 1 and are constant variables. The difference between two adjacent levels 
is the same and is equal to $V_{d c}$. Each full-bridge inverter produces a three-level waveform $\left(+V_{d c},-V_{d \mathrm{c}}\right.$, and 0$)$, and each angle $\theta_{i}$ is related to inverter $i$, where $i=1,2, \ldots, S$. S is the number of DC sources that is equal to the number of switching angles (in this study, $S=\emptyset$ ). The number of levels (L) is calculated as $L=2 S+1$.

Considering equal amplitude of all DC sources, the Fourier series expansion of the output voltage waveform is

$$
V(\omega t)=\sum_{n=1}^{\infty} V_{n} \sin (n \omega t)
$$

where $V_{n}$ is the amplitude of harmonics. The angles are limited between $0^{\circ}$ and $90^{\circ}(0 \leq \theta \leq 90)$. Due to odd quarterwave symmetric characteristics, the harmonics with even order are 0 . Consequently, $V_{n}$ becomes

$$
V_{n}=\left\{\begin{array}{lr}
\frac{4 V_{d c}}{n \pi} \sum_{i=1}^{k} \cos \left(n \theta_{i}\right) & \text { for odd } n \\
0 & \text { for even } n
\end{array}\right.
$$

The two approaches used for adjusting the switching angles are as follows:

1. Minimizing total harmonic distortion (THD), which is not common because some low order harmonics may remain.

2. Canceling the lower order harmonics and removing the remaining harmonics with a filter.

The second approach is preferred. For motor drive applications, it is necessary to eliminate low order harmonics from 5 to 17 . For this purpose, in this paper, a 13-level inverter is chosen to eliminate low harmonics (i.e., from 5 to 17). Deleting triple harmonics is unnecessary because they are eliminated automatically in the three-phase output voltage. For a 13-level inverter, Eq. (2) results in Eq. (3).

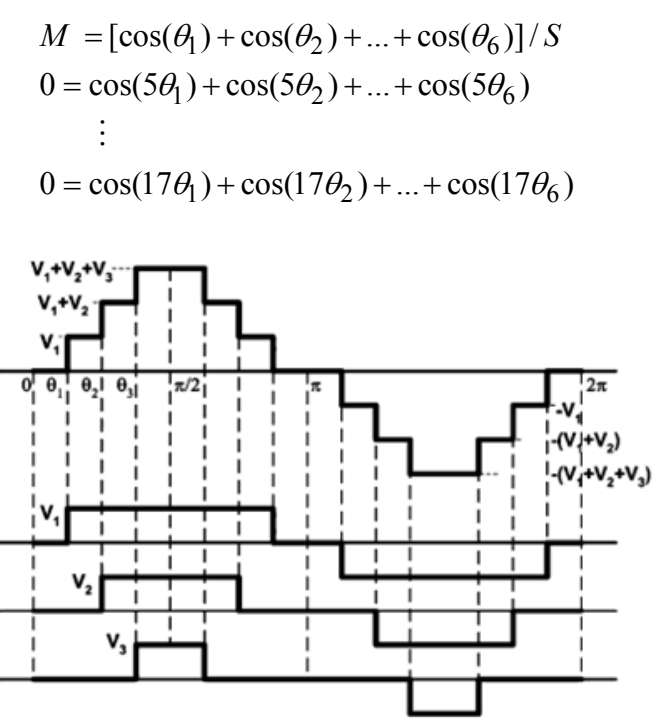

Fig. 3. A seven-level inverter. where $M$ is modulation index defined as

$$
M \stackrel{\Delta}{=} \frac{V_{1}}{4 V_{d c} S / \pi} \quad(0<M \leq 1)
$$

Determining the six switching angles, namely, $\theta_{1}, \theta_{2}, \theta_{3}$, $\theta_{4}, \theta_{5}$, and $\theta_{6}$, is required to satisfy Eq. (3). All in this set equation is nonlinear, and different solution methods can be applied. In the next section, BFA is employed to determine the proper switching angles. Finally, the comparison between BFA and GA is presented.

\section{Bacterial Foraging Algorithm}

Recently, a new algorithm called BFA has been used for solving optimization problems. BFA is based on the premise that natural choices have the tendency to eliminate animals with poor talent in searching for food and favor those employing successful strategies. After many experiments and generations, the poor strategies for searching food are either canceled or revised.

The bacterial foraging system consists of four principal mechanisms, namely, chemotaxis, swarming, reproduction, and elimination-dispersal [19-24]. A brief description of each of these processes is presented below.

\subsection{Chemotaxis}

This process simulates the movement of an E. coli cell through swimming and tumbling via flagella. An $E$. coli bacterium can move in two different ways. It can swim for a time-period in the same direction or it may tumble, and alternately move between these two modes of operation for its entire lifetime. Suppose $\theta^{i}(j, k, l)$ represents $i$-th bacterium at $j$-th chemotactic, $k$-th reproductive, and $l$-th elimination-dispersal step, and $C(i)$ is the size of the step taken in the random direction specified by the tumble (run length unit). In computational chemotaxis, the movement of the bacterium may be represented by

$$
\theta^{i}(j+1, k, l)=\theta^{i}(j, k, l)+C(i) \frac{\Delta(i)}{\sqrt{\Delta^{T}(i)^{*} \Delta(i)}}
$$

where $\Delta$ denotes a vector in the random direction whose elements lie in $[-1,1]$.

We can then define Eq. (6):

$$
\begin{aligned}
& \varphi(i)=\frac{\Delta(i)}{\sqrt{\Delta^{T}(i)^{*} \Delta(i)}}, \\
& \theta^{i}(j+1, k, l)=\theta^{i}(j, k, l)+C(i) \varphi(i)
\end{aligned}
$$

To represent a tumble, a unit length random direction, $\varphi(\mathrm{i})$, is generated. This is then used to define the direction of movement after a tumble. 


\subsection{Swarming}

It is always desired for the bacterium, which has searched the optimum path of food, to attract other bacteria in order to reach the desired place more rapidly. Swarming makes the bacteria congregate into groups and hence, move as concentric patterns of groups with high bacterial density. Mathematically, swarming can be represented by

$$
\begin{aligned}
J_{c c}(\theta, P(i, j, l)) & =\sum_{i=1}^{S} J_{c c}^{i}\left(\theta, \theta^{i}(j, k, l)\right) \\
& =\sum_{i=1}^{S}\left[-d_{\text {atract }} \exp \left(-\omega_{\text {atract }} \sum_{m=1}^{p}\left(\theta_{m}-\theta_{m}^{i}\right)^{2}\right)\right] \\
& +\sum_{i=1}^{S}\left[-h_{\text {repelent }} \exp \left(-\omega_{\text {repelent }} \sum_{m=1}^{p}\left(\theta_{m}-\theta_{m}^{i}\right)^{2}\right)\right]
\end{aligned}
$$

where $J_{C C}(\theta, P(j, k, l))$ is the cost function value added to the actual cost function that would be minimized. This then presents a time varying cost function. $S$ is the total number of bacteria; $p$ is the number of parameters to be optimized, which are presented in each bacterium; $d_{\text {attract }}, \omega_{\text {attract }}, h_{\text {repe- }}$ lent, and $\omega_{\text {repelent }}$ are the different coefficients to be chosen cautiously.

\subsection{Reproduction}

The least healthy bacteria eventually die, whereas each of the healthier bacteria (i.e., those yielding the lower value of the objective function) asexually split into two, which are then placed in the same location. This keeps the swarm size constant.

\subsection{Elimination and Dispersal}

In the local environment, the life of a population of bacteria changes either gradually by consumption of nutrients or suddenly due to some other influence. Events can kill or disperse all the bacteria in a region. They have the effect of possibly destroying the chemotactic progress; however, they also assist such processes, as dispersal may place bacteria near good food sources. Elimination and dispersal help reduce the behavior brought about by stagnation (i.e., being trapped in a premature solution point or local optima).

\section{Implementation}

In this section, the BFA program is written by using a MATLAB software in order to show the advantages of the proposed algorithm. The fitness function and its limitations are shown in Eqs. (8) and (9), respectively.

$$
f=\min _{\theta_{i}}\left\{\left(100 \frac{V_{1}^{*}-V_{1}}{V_{1}^{*}}\right)^{4}+\sum_{s=2}^{S} \frac{1}{h_{s}}\left(50 \frac{V_{h_{s}}}{V_{1}}\right)^{2}\right\} ; i=1,2, \ldots, S
$$

which is subject to

$$
0 \leq \theta_{i} \leq \pi / 2
$$

The results of running this program are shown after implementing a 13-level inverter as the case study in order to eliminate its low order harmonics, i.e., from $5^{\text {th }}$ to $17^{\text {th }}$. Fig. 4 shows the amount of fitness function for running the program once and 10 times. The step of variation of the modulation index $(M)$ is 0.01 . Since this program targets optimization, for some values of $M$ wherein the functions of Eq. (3) offer feasible answers, the program can correspondingly arrive at successful solutions. However, in other $M \mathrm{~s}$, solutions are obtained based on the lowest amount of fitness function. The probability of converging to global minima is greater for programs run 10 times compared with those run at a single time.

The vertical axis depicts the optimum objective value. A value less than $10^{-2}$ indicates that the program has reached global minima. For other values of the fitness function, solutions might not be achieved at all; that is, the algorithm has converged in local minima. To illustrate the effect of the number of runs, Fig. 5 is shown; herein, the probability of cumulative distribution function (CDF) of the fitness function for 1-, 2-, 5-, and 10-time runs times is shown. This figure also shows that the probability of obtaining the fitness function value below $10^{-5}$, after $1-, 2-, 5-$, and $10-$ time runs is $19.8 \%, 31.2 \%, 34.4 \%$, and $39.8 \%$, respectively.

For some modulation indices, more than one solution may exist. The program sometimes encounters one of them. Fig. 6 shows the situation of switching angles versus M. In Fig. 7, the results of harmonic conditions and the THD value are shown. For the region at which the fitness function value is low, all low order harmonics are maintained close to 0 . In other ranges, the value of harmonics is significant, which means that in this range of solution region, the equations cannot be solved.

Inasmuch as the penalty considered for satisfying the desired fundamental harmonic is high, this harmonic approaches the desired value in the output of the inverter, even in the region where the equations do not have feasible solutions.

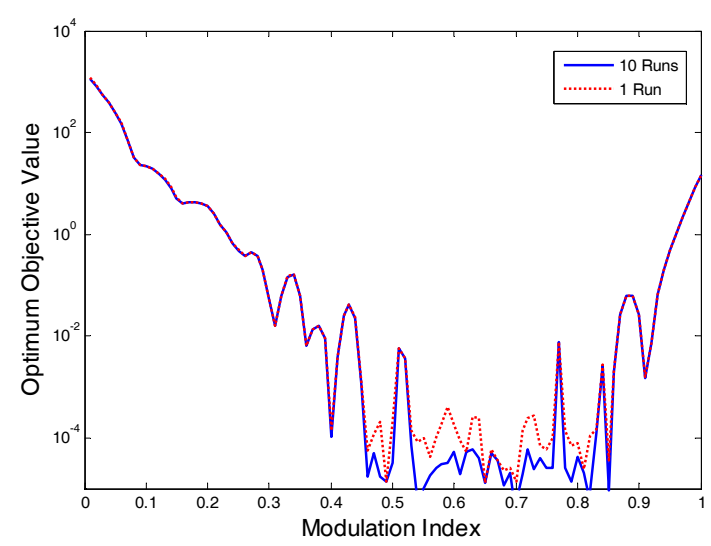

Fig. 4. Optimum value of the objective function versus $M$ for the program in 1- and 10-time runs. 


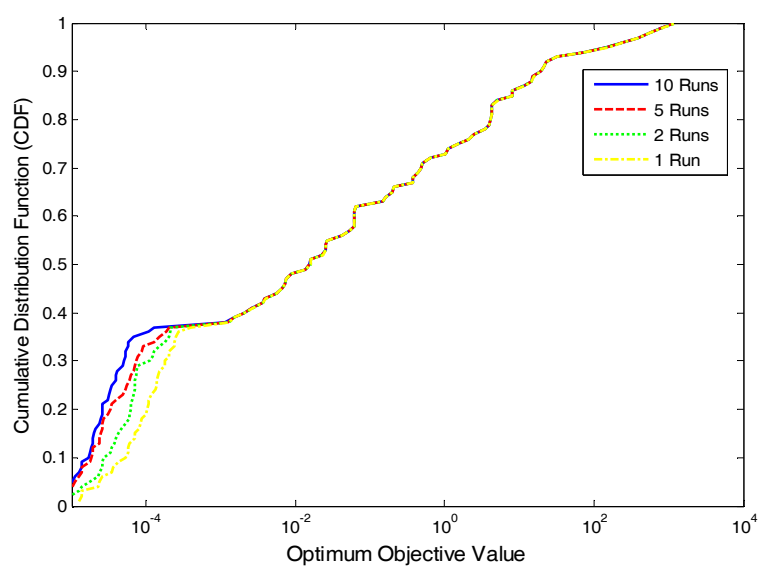

Fig. 5. CDF of the optimum objective function for a 13level inverter over its $\mathrm{M}$ range.

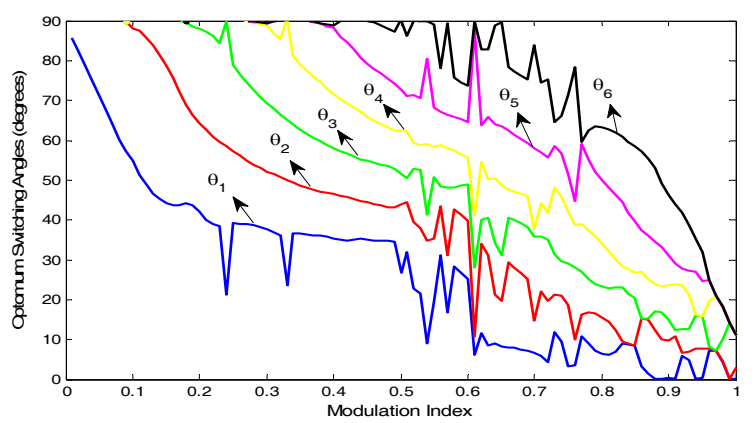

Fig. 6. Optimal switching angles versus $M$.

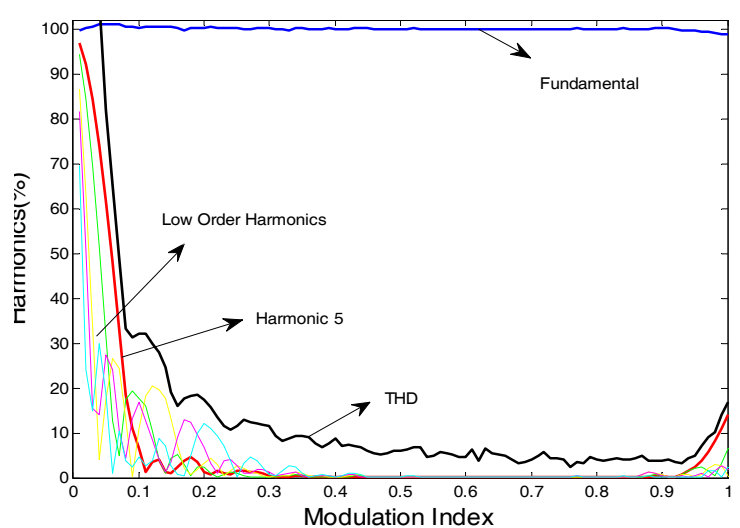

Fig. 7. Percentages of fundamental and low order harmonics and THD.

The value of THD is treated similar to that of low order harmonics. The reduction in value of low order harmonics leads to a decrease in THD value.

To verify the results of BFA, comparisons are made between BFA and GA. GA is the most well known intelligent algorithm. Fig. 8 compares the CDF of BFA and GA in one-time running. It also illustrates BFA's advantages over GA, and shows that the probability in BFA to reach global optima is more likely than that of GA. Figs. 8-11 show the CDFs for 2-, 5-, and 10-time runs. These figures also show the advantage of BFA over GA.

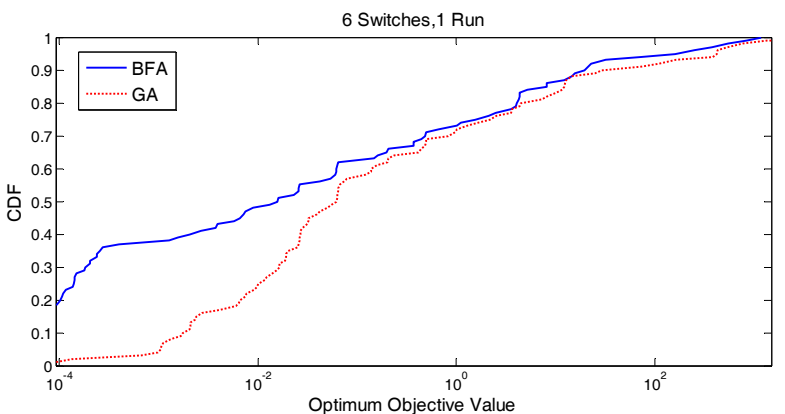

Fig. 8. Comparison of BFA and GA for 17-level inverters in 1-time running.

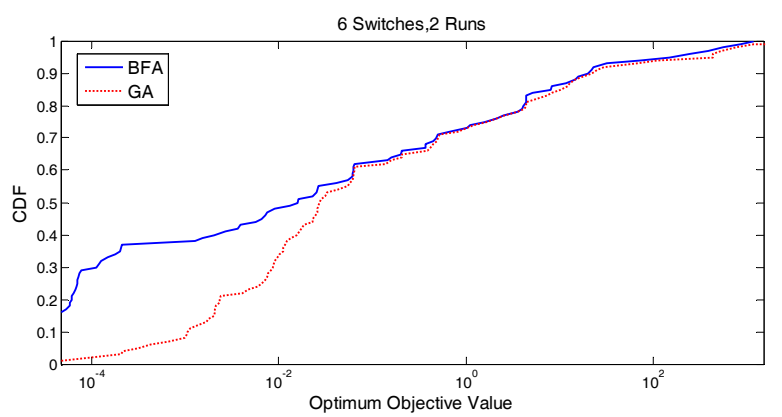

Fig. 9. Comparison of BFA and GA for 17-level inverters in 2- time runs.

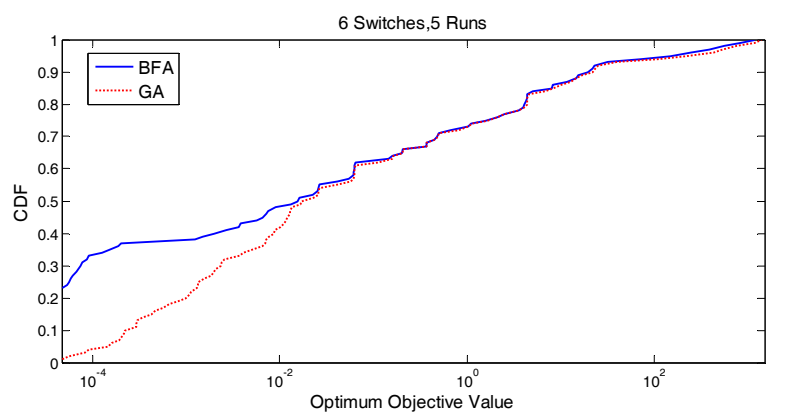

Fig. 10. Comparison of BFA and GA for 17-level inverters in 5-time runs.

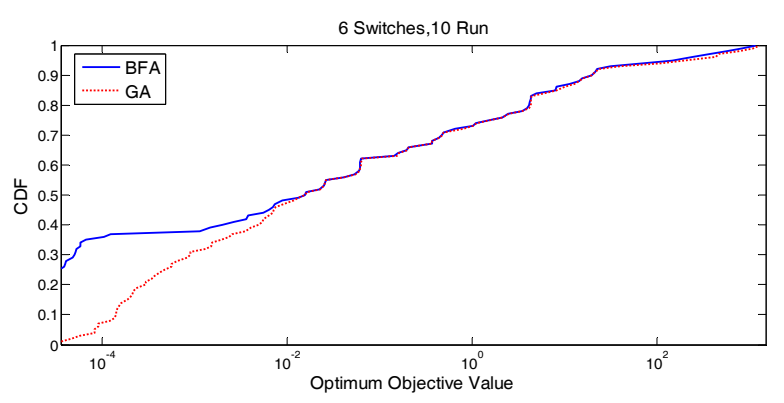

Fig. 11. Comparison of BFA and GA for 17-level inverters in 10-time running.

\section{Conclusion}

The goal of this paper is to eliminate low order harmonics 
using the OHSW technique. The proposed method (i.e., BFA) was used to find solutions in nonlinear equations. Simulation results show that harmonics have been successfully omitted. However, at some operating points, the harmonics cannot be removed and thus should be minimized. Based on the comparison of BFA and GA, the former yields a better solution and with higher probability in reaching global minima.

\section{References}

[1] J. Rodriguez, Jih-Sheng Lai, and Fang Zheng Peng. "Multilevel inverters: a survey of topologies, controls, and applications" IEEE Transactions on Industrial Electronics, Volume 49, Issue 4, Aug. 2002 Page(s): 724-738.

[2] Farokhnia, N., Fathi, S.H., and Toodeji, H.R. "Direct Nonlinear Control for Individual DC Voltage Balancing in Cascaded Multilevel DSTATCOM" IEEE International Conference on Electric Power and Energy Conversion Systems (EPECS) 2009, Publication Year: 2009, Page(s): 1-8.

[3] Flourentzou, N., Agelidis, V.G., and Demetriades, G.D. "VSC-Based HVDC Power Transmission Systems: An Overview" IEEE Transactions on Power Electronics, Volume 24, Issue 3, March 2009 Page(s): 592-602.

[4] Rodriguez, J., Bernet, S., Bin Wu, Pontt, J.O., and Kouro, S. "Multilevel Voltage-Source-Converter Topologies for Industrial Medium-Voltage Drives" IEEE Transactions on Industrial Electronics, Volume 54, Issue 6, Dec. 2007 Page(s):2930-2945.

[5] Kaviani, A., Fathi, S.H., Farokhnia, N., and Ardakani, A. "PSO, an effective tool for harmonics elimination and optimization in multilevel inverters" 4th IEEE Conference on Industrial Electronics and Applications, 2009. ICIEA 2009. 25-27 May 2009, Page(s): 2902-2907.

[6] Yousefpoor, N., Farokhnia, N., Fathi, S.H., and Moghani, J. "Application of OHSW Technique in Cascaded Multilevel Inverter with Adjustable DC Sources" IEEE International Conference on Electric Power and Energy Conversion Systems (EPECS) 2009, Publication Year: 2009, Page(s):1-6.

[7] Yousefpoor, N., Farokhnia, N., Fathi, S.H., and Moghani, J. "Developed Single-Phase OMTHD Technique for Cascaded Multilevel Inverter By Considering Adjustable DC Sources" IEEE International Conference on Electric Power and Energy Conversion Systems (EPECS) 2009, Publication Year: 2009, Page(s):1-6.

[8] Barkati, S., Baghli, L., Berkouk, E.M, and Boucherit, M. Harmonic elimination in diode-clamped multilevel inverter using evolutionary algorithms. Electr. Power Syst. Res. (2008), doi:10.1016/j.epsr.2008.03.010.

[9] McGrath, B.P. and Holmes, D.G. "Natural Capacitor Voltage Balancing for a Flying Capacitor Converter
Induction Motor Drive" IEEE Transactions on Power Electronics, Volume 24, Issue 6, June 2009 Page(s): 1554-1561.

[10] Zhong, Du, Tolbert, L.M., Ozpineci, B., and Chiasson, J.N. "Fundamental Frequency Switching Strategies of a Seven-Level Hybrid Cascaded H-Bridge Multilevel Inverter" IEEE Transactions on Power Electronics, Volume 24, Issue 1, Jan. 2009 Page(s):25-33.

[11] Akbari, H. and Gharehpetian, G.B. "Harmonic Distortion Minimization in Multilevel Converters for a Wide Range of Modulation Indexes" IEEE International Aegean Conference on Electrical Machines and Power Electronics 2007 ACEMP '07, 10-12 Sept. 2007 Page(s):581-586.

[12] El-Naggar, K. and Abdelhamid, T.H. "Selective harmonic elimination of new family of multilevel inverters using genetic algorithms," Energy Conversion and Management, Volume 49, Issue 1, January 2008, Pages 89-95.

[13] Tianhao, Tang, Jingang, Han, and Xinyuan, Tan. "Selective Harmonic Elimination for a Cascade Multilevel Inverter,” 2006 IEEE International Symposium on Industrial Electronics, Vol. 2, July 2006 Page(s): 977-981.

[14] Zhong, Du, Tolbert, L.M, Chiasson, J.N, and Ozpineci, B. "Reduced Switching-Frequency Active Harmonic Elimination for Multilevel Converters," IEEE Transactions on Industrial Electronics, Volume 55, Issue 4, April 2008 Page(s):1761-1770.

[15] Ozpineci, B, Tolbert, L.M, and Chiasson, J.N. "Harmonic optimization of multilevel converters using genetic algorithms," IEEE Power Electronics Letters, Volume 3, Issue 3, Sept. 2005 Page(s):92-95.

[16] Hosseini-Aghdam, M.G, Fathi, S.H, and Gharehpetian, G.B "Elimination of Harmonics in a Multilevel Inverter with Unequal DC Sources Using the Homotopy Algorithm," IEEE International Symposium on Industrial Electronics, 2007, ISIE 2007, 4-7 June 2007 Page(s):578-583.

[17] Barkati, S., Baghli, L., Berkouk, E.M, and Boucherit, M. "Harmonic elimination in diode-clamped multilevel inverter using evolutionary algorithms," Electr. Power Syst. Res. (2008), doi:10.1016/j.epsr.2008.03. 010.

[18] Sirisukprasert, Siriroj. "Optimized Harmonic SteppedWaveform for Multilevel Inverter", Msc thesis, 1999 available at http://www.worldcatlibraries.org.

[19] Passino, K. M. "Biomimicry of bacterial foraging for distributed optimization and control" IEEE Control System Magazine, Volume 22, Issue 3, Publication Year: 2002, Page(s):52- 67.

[20] Senjyu, T., Yamashiro, H., Shimabukuro, K., Uezato, K., and Funabashi, T. "Fast solution technique for large-scale unit commitment problem using genetic algorithm" IEE Proceeding to Generation, Transmission and Distribution, Volume: 150, Issue: 6, Publication Year: 2003, Page(s):753-760.

[21] Mishra, S. "A hybrid least square-fuzzy bacteria for- 
aging strategy for harmonic estimation" IEEE Transactions on Evolutionary Computation, Volume: 9, Issue: 1, Publication Year: 2005, Page(s):61-73.

[22] Eslamian, M., Hosseinian, S. H., and Vahidi, B. "Bacterial Foraging-Based Solution to the UnitCommitment Problem," IEEE Transactions on Power Systems, Volume 24, Publication Year: 2009, Page(s): 1478-1488

[23] Tripathy, M. and Mishra, S. "Bacteria foraging-based solution to optimize both real power loss and voltage stability limit," IEEE Transactions on Power Systems, Volume 22, Publication Year: 2007, Page(s):240-248.

[24] Kim, D. H., Abraham, A., and Cho, J. H. "A hybrid genetic algorithm and bacterial foraging approach for global optimization" Elsevier, An International Journal of Information Sciences, Volume 177, Issue 18, 15 September 2007, Pages 3918-3937. 